\section{Adrenoleukodystrophy linked to Chromosome $X$ with Neuropsychiatric Symptoms and Frontal Involvement: Case Report}

\section{Abstract}

Leukodystrophies are a rare group of genetic disorders that affect white matter of the central and peripheral nervous system. We present a clinical case of X-linked adrenoleukodystrophy (X-ALD) in a 9-year old male patient. The patient initially presented with neuropsychiatric symptoms characterized by impulsiveness, hyperactivity, and loss of reading and writing abilities. MRI scan showed a frontal involvement of both cerebral hemispheres. Based on this experience, we recommend that patients with hyperactivity and impulsiveness, and loss of previously acquired abilities such as reading and writing should be thoroughly evaluated to rule out the presence of X-ALD. After making the diagnosis with a complete serum fatty acid profile accompanied by the radiologic findings aforementioned, the patient underwent bone marrow transplant, without a favorable outcome after one year of follow-up.

Keywords: Adrenoleukodystrophy; X chromosome; White matter; Adrenal insufficiency; ADHD
Sergio Abraham Lozano Garza1*, Santos-Guzmán', Arturo Garza Peña ${ }^{2}$, Raúl Calderón², Jesús and Carlos Aguirre Velázquez ${ }^{2}$

1 Tecnológico de Monterrey, Escuela de Medicina y Ciencias de la Salud

2 Pediatric Neurology, Tecnologico de Monterrey Escuela de Medicina y Ciencias de la Salud, Mexico

3 Tecnologico de Monterrey, Escuela de Medicina y Ciencias de la Salud, Mexico *Corresponding author: Sergio Abraham
Lozano Garza

lozano.sergio89@gmail.com

Tecnológico de Monterrey, Escuela de Medicina y Ciencias de la Salud.

Tel: +528119823507

Citation: Garza SAL, Peña AG, Calderón R, Santos-Guzmán J, Velázquez CA (2019) Adrenoleukodystrophy linked to Chromosome $X$ with Neuropsychiatric Symptoms and Frontal Involvement: Case Report. J Child Dev Disord. Vol.5 No.2:7

11 months old brother has a learning disorder based primarily on reading. The patient's perinatal history was unremarkable, he was born after a full term pregnancy, by vaginal delivery with no obstetric trauma, natural birth, cries and breathes upon birth weighting 3,000 grams measuring $50 \mathrm{~cm}\left(25^{\text {th }}\right.$ and $50^{\text {th }}$ percentile, respectively). Apgar score of 9-9. The assessment of neurodevelopment was performed clinically, no scales were used.

Symptomatology began at 6 years old, being incapable of paying attention at simple tasks while performing physical activities at school, also impulsiveness and hyperactivity symptoms, with difficulty to perform academic work. After that, the patient suffers from loss of reading and writing capabilities. The patient's parents turn to psychology, where neuropsychological tests were applied
A 9-year old male patient was referred to our clinic. Past medical history included a maternal uncle's decease from an unspecified neurological disease at 40 years of age. The patient's 5-years and 
obtaining a maturity of age 6 and an intellectual coefficient of 68 , and the patient received cognitive-behavioral therapy for 6 months. The patient did not show no clinical improvement and emotional liability and dyspraxia were shown: bradylalia later appears in normal speech, as well as loss of sphincter control.

He was seen by a pediatric neurologist and based on interrogatory and physical, diagnosed attention deficit and hyperactivity disorder. He received methylphenidate at scaling dosages of 5 $\mathrm{mg}$ daily before school and rising up to $10 \mathrm{mg}$ daily before school with no improvement, this therapeutic regimen was given for approximately a year. The patient showed no improvement at all and continued with slow and slurred speech and emotional ability, no other tests were performed ant the parents were told that this was a behavioral issue only.

At 7 years-old the patient was admitted to the emergency department due to an epileptic seizure. MRI scan showed the involvement of frontal white matter (Figure 1a and 1b), and begun oral treatment with Levetiracetam at a total dose of 25 $\mathrm{mg} / \mathrm{kg}$ of weight daily in 2 doses per day.

Due to the findings on the MRI scan, a fatty acid profile in serum was ordered (Table 1 ) and the diagnosis of X-ALD was made. No other event was recorded until he underwent a bone marrow transplant at 8 years old and later the placement of a feeding tube that same year.

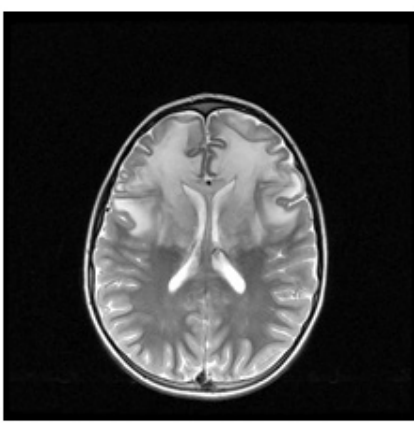

a

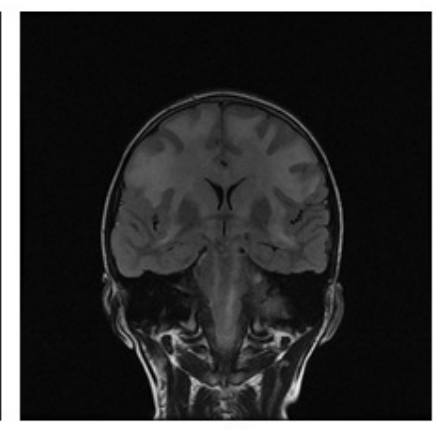

$\mathrm{b}$
Figure 1 ( $a$ and $b$ ) A clear involvement of white substance can be appreciated in the axial and coronal cortex views.

Table 1 The results of the patient's profile of fatty acids in plasma are shown.

\begin{tabular}{|c|c|c|}
\hline \multicolumn{3}{|c|}{ Fatty Acids Profile } \\
\hline C22:0 & $56 \mathrm{nmol} / \mathrm{mL}$ & $<96.3$ \\
\hline C24:0 & $90.5 \mathrm{nmol} / \mathrm{mL}$ & $<91.4$ \\
\hline C26:0 & $3.85 \mathrm{nmol} / \mathrm{mL}$ & $<1.30$ \\
\hline C24:0/C22:0 & $1.62 \mathrm{ratio}$ & $<1.39$ \\
\hline C26:0/C22:0 & $0.069 \mathrm{ratio}$ & $<0.023$ \\
\hline Pristanic acid & $0.06 \mathrm{nmol} / \mathrm{mL}$ & $<2.98$ \\
\hline Phytanic acid & $0.85 \mathrm{nmol} / \mathrm{mL}$ & $<9.88$ \\
\hline Pristanic/Phyanic & $0.07 \mathrm{ratio}$ & $<0.39$ \\
\hline
\end{tabular}

The patient turns to pediatric neurology at 7 years old, and a 30-minute electroencephalogram with sleep suppression was indicated. This study was reported as normal (Figure 2). Combined type attention deficit disorder and hyperactivity was diagnosed. Initial oral treatment with methylphenidate, $10 \mathrm{mg}$ qd was recommended. Despite this management, neurological symptoms progressed and his academic performance deteriorated. At the age of 7 he showed abnormal movements compatible with seizures, characterized by right cephalic version and sight aversion on the same side, with an increase in muscular tone in the right half of the body. The patient was submitted to bone marrow transplant at 8 years old a recommended procedure for this conditions [3].

He later showed abnormal movements characterized by the extension of the left chest limb and flexion of the right chest limb, particularly upon exaltation or stress. Such movements stop with rest and sleep. Physical exploration showed increase in pigmentation, particularly in the bilateral underarm areas and bilateral groin areas were found. The total affectation of superior mental functions was present, and the patient does not emit speech. Preserved state of consciousness was noted throughout the physical exam. Physical exploration revealed cranial nerve alteration in $\mathrm{V}, \mathrm{IX}, \mathrm{X}$ with chewing and swallowing problems, generalized hyperreflexia with patellar right clonus, a notorious increase in muscular tone with hypotrophy of the limbs. The limb strength was not possible to asses. Patient had a positive reaction to nociceptive stimuli with poor muscular contraction; limb coordination was non-examinable. Positive Babinski reflex on both limbs. The patient exhibits dystonic movements of the right arm on flexion and left arm extension, which are more evident during neurological exploration (while experiencing an episode of stress due to medical exploration). Dystonic movements of infancy beginning (2-12 years) were considered segmentary, paroxistic and associated to other neurological signs of degenerative etiology, with hereditary and familiar patterns $[4,5]$.

The patient was treated with clonazepam, baclofen and trihexyphenydil for the control of these movements. It should be mentioned that the patient is under treatment from endocrinology with steroids to control adrenal insufficiency. The patient is unable to walk, moves in a wheelchair. He receives 4 drugs regimen (clonazepam, baclofen, trihexyphenidyl and levetiracetam). It is noted that these dystonic movements increased in periods of stress and were notably diminished with sleep and rest. The patient has a seizure pattern of approximately 2 events per month, of short duration (less than 20 seconds most of them). We will continue seeing the patient to adjust dosages, with the final intention to improve this child's quality of life given the fact that he did not improve after the bone marrow transplant (which must be done in early stages of the disease). 


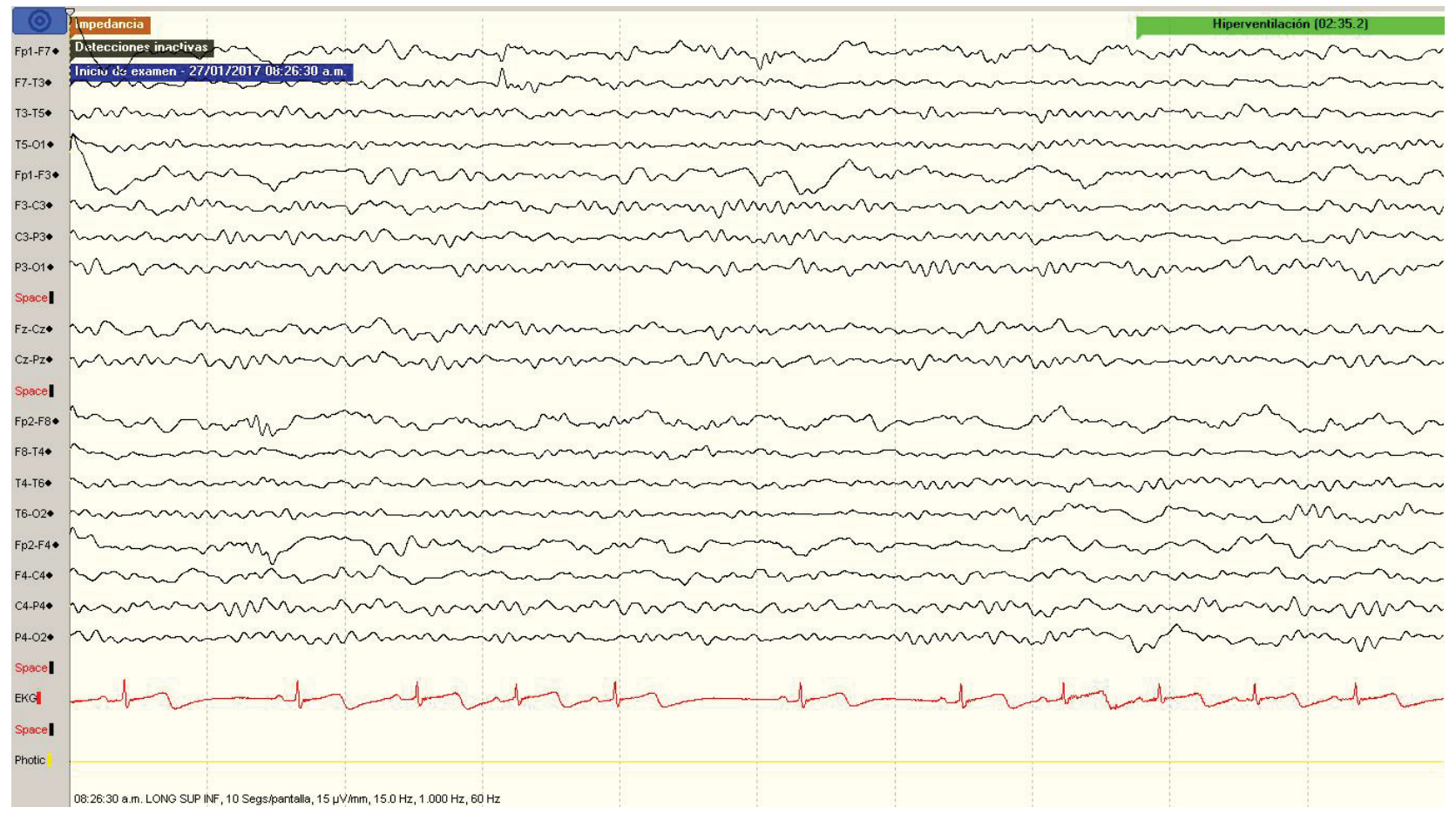

Figure 2 EEG performed according to 10-20 international classification. Patient with sleep suppression. A base alpha rhythm can be appreciated in the rear, and a bioelectric gradient is moderately integrated. No abnormal EEG phenomena were observed during the course of the study.

\section{Discussion and Conclusion}

Adrenoleukodystrophy linked to $X$ is a demyelinating disorder that significantly deteriorates the patient's quality of life. There is an alteration in the metabolism of very long-chain fatty acids that makes them accumulate in plasma and tissue. These acids are distinguished by having more than 22 carbons in their chain. This disorder occurs in approximately $1 / 17000-20000$ males and is responsible for approximately $10 \%$ of adrenal insufficiency in males. Radiologically, the parieto-occipital area can be seen affected in $85 \%$ of cases, and the frontal area in $15 \%$ of the cases. The defect is found in chromosome $\mathrm{Xq28}$, which is not able to codify the ABDC1 gene correctly. More than 600 mutations have been described and the phenotype has no relation to the mutation. This gene codifies the peroxisomal transmembrane protein (ALDP) that is involved in the transport of COA-VLCFA esters from the cytosol to the peroxisome, which gives way to the decrease in the degradation of very long-chain saturated acids and results in the accumulation of such fatty acids. The accumulation of VLCFA in the glial cells produces a destabilization of the myelin sheath and the deterioration of axonal function [6]. As previously mentioned, there are a considerable variety of phenotypes: infantile cerebral, at the beginning of adolescence, at the beginning of adulthood, adrenomyeloneuropathy with and without cerebral involvement, pure adrenal insufficiency and women with adrenoleukodystrophy. The most common of these types is the cerebral infantile type. It appears between ages 2.5 and 10 [7]. It begins with neuropsychiatric symptoms, such as emotional liability, hyperactivity and impulsiveness. It is frequently confused with attention deficit and hyperactivity disorders of any subtype, which causes a delay in the correct diagnosis. In this patient those were the initial symptoms, since the frontal lobes - where the prefrontal, dorsolateral, ventromedial and orbitofrontal cortex is found - in charge of processing emotions, inhibiting inappropriate behaviors and superior mental functions were primarily affected [8]. The patient may subsequently exhibit convulsive crises, although this is not entirely common since initially the white matter is affected, and later gray matter. With the progression of the disease, the patient shows heterogeneous neurological symptomatology (spasticity, dystonia, loss of speech, swallowing alterations, among others) and deteriorates rapidly. Death usually occurs approximately 3 years after diagnosis if not detected and treated rapidly [2].

The treatment of choice for these patients is typically a bone marrow transplant (BMT). To this date, no success rates for patients with BMT for ALD have been found. The BMT may stop the progression of the disease as long as it is performed at an early stage and with none or very few neuropsychiatric alterations on the patient. A Loes's score (radiological index to stage the degree of affection to the central neural system) of less than 9 and an intellectual coefficient greater than 80 are recommended to prognosticate a favorable outcome in this therapeutic modality [9]. Diet therapy with Lorenzo's oil (monounsaturated fatty acids) has also been proposed. However, it has not been proven that they work to prevent the symptoms, much less revert them [10]. It is a fact that this condition continues to be a challenge for any 
specialist, both in the aspect of considering the initial diagnosis as well as in opportune treatment. Research on a greater scale towards new therapeutic modalities should be encouraged, in order to improve the quality of life of our patients.

\section{References}

1 Palmero OE, Cruz INP, Sardañas SS, Cepero MTV (2014) Adrenoleukodystrophy X. A case report. Hospital General Docente “Roberto Rodriguez Fernandez" Morón. Mediciego 20: 1.

2 Mingorance Delgado A, Tabernero Pérez C, Tapia Muñoz J, Martín Aguadi MJ, Lloret Sempere T, et al. (1999) Adrenoleucodistrofia ligada al cromosoma X. A propósito de un nuevo caso. An Esp Pediatr 50: 509-512.

3 Suzuki $Y$, Isogai $K$, Teramoto $T$, Tashita $H$, Shimozawa $N$, et al. (2000) Bone marrow transplantation for the treatment of X-linked Adrenoleukodystrophy. J Inherit Metab Dis 23: 453-458.

4 Jinnah HA, Albanese A (2014) The new classification system for the dystonias: Why was it needed and how was it developed? Mov Disord Clin Pract 1: 280-284.

5 Abdo WF, Van De Warrenburg BPC, Burn DJ, Quinn NP, Bloem BR (2010) The clinical approach to movement disorders. Nat Rev Neurol 6: 29-37.

\section{Acknowledgment}

We thank the important contribution of Dr. Daniel Olvera Posada, a Writing Lab mentor for the his editorial review and comments.

6 Úbeda ML, de Arriba Muñoz A, Lozano MF, Aizpún JIL, Jiménez MCG (2017) Adrenoleucodistrofia ligada al X: presentación de tres casos. Importancia del diagnóstico precoz. Arch Argent Pediatr 115: e279-e281.

7 Engelen M, Kemp S, Visser M De, Geel BM Van, Wanders RJA, et al. (2012) X-linked adrenoleukodystrophy (X-ALD): clinical presentation and guidelines for diagnosis, follow-up and management. Orphanet J Rare Dis 7: 51.

8 Flores Lázaro JC, Ostrosky-Solís F (2008) Neuropsicología de Lóbulos Frontales, Funciones Ejecutivas y Conducta Humana. Revista Neuropsicología, Neuropsiquiatría y Neurociencias 8: 47-58.

9 Miller W (2017) Stem cell-transplantation therapy for adrenoleukodystrophy: current perspectives. J Neurorestoratol 5 : 5-19.

10 Pellicer JG, Boquet EM, Cabanas JG, Pia PC (1996) Aceite De Lorenzo En El Tratamiento De La Adrenoleucodistrofia: Esperanza O Realidad? Farm Hosp 20: 1-7. 